\title{
Proceeding
}

Supplementary Issue: Summer Conferences of Sports Science. Costa Blanca Sports Science Events, 20-21 September 2019.

Alicante, Spain.

\section{Study on correlation between motor and memory learning}

\author{
DAVIDE DI PALMA ${ }^{1}$, FRANCESCO PERROTTA², DOMENICO TAFURI ${ }^{1}$ \\ ${ }^{1}$ Parthenope University, Naples, Italy \\ 2M.I.U.R. Campania, Italy
}

\begin{abstract}
The Knowledge of the Result for motor learning, relationship between subjective estimation of the error and relative frequency of external feedback. Research on the learning of a motor task, although generally conducted on healthy individuals, can offer useful indications about the best strategies to be adopted in the rehabilitation of subjects with CNS lesions. In fact, rehabilitation can be considered as a learning process in pathological conditions. There are numerous experimental evidences that, a lower relative frequency (FR) with which it is provided to the one who learns the knowledge of the result (CR) about the outcome of the response, and the request of the formulation of a subjective estimate before the CR (SS) both positively affect the fixation of the motor task. Recently, however, the possibility of an interaction between these two variables has been suggested, in the sense that the subject, when he must formulate a subjective estimate of the error, would benefit from a greater, and not a lesser, FR. To verify this, 60 healthy young subjects (mean age 24.1 \pm 3.2 ) performed a simple task of producing a concentric work target with flexed elbow muscles during isokinetic contraction at a rate of 30 degrees / second. During the practice trials, subjects a) were required, or were not required, to estimate the error made in the newly concluded trial, and b) $C R$ was provided after each trial $(100 \% \mathrm{FR})$ or after one in every five trials ( $20 \% \mathrm{FR})$. To further stress the difference between the subjects who were or should not formulate an SS, the latter was asked, immediately after the conclusion of the trial, to perform a simple mental calculation. All subjects performed 15 sets of 10 repetitions of the task during a single practice session. A retention test (1 set of 10 repetitions without CR or SS) was performed the following day. The comparison between the groups in the retention test was performed with the analysis of the variance, before and after adjustment for the initial conditions. The results showed that, after adjusting for the initial conditions, the group of subjects who received CR with $100 \% \mathrm{FR}$ and who had to formulate the SS during the practice period, performed the retention test in a significantly better way. Keywords: Attention; Memory; Motor learning.
\end{abstract}

\section{Cite this article as:}

Di Palma, D., Perrotta, F., \& Tafuri, D. (2019). Study on correlation between motor and memory learning. Journal of Human Sport and Exercise, 14(5proc), S1950-S1962. doi:https://doi.org/10.14198/ihse.2019.14.Proc5.15

Corresponding author. Parthenope University, Naples, Italy.

E-mail: domenico.tafuri@uniparthenope.it

Supplementary Issue: Summer Conferences of Sports Science. Costa Blanca Sports Science Events, 20-21 September 2019. Alicante, Spain.

JOURNAL OF HUMAN SPORT \& EXERCISE ISSN 1988-5202

(c) Faculty of Education. University of Alicante

doi:10.14198/jhse.2019.14.Proc5.15 


\section{INTRODUCTION}

When talking about "learning" indicates a "relatively durable modification induced in an organism directly from experience"; so, we are in front of a complex process that involves various aspects of interacting with each other and whose distinctive stability over time. In fact, the relatively fixed modification in the behaviour is the thing that differs from the temporary improvement of learning performance. If the next day you are not able to remember how to perform the task, it has not been learned. To assess whether it is transient changes or real learning, is necessary so that the performance is evaluated again later. It was introduced by the researchers an experimental protocol can distinguish between temporary effects (performance improvement) and relatively permanent effects (learning), which has two phases (Winstein, 1991):

1) The acquisition phase (or practice), during which the subject performs numerous trials (trial); at this stage the different experimental groups receiving treatment with different levels of the independent variable in question, for example, different extrinsic feedback programs;

2) Transfer phase, in which all groups are transferred to a common level of the studied variable, such as the absence of extrinsic feedback; this phase must be sufficiently separated in time from the stage of practice, so that the transient effects of the independent variable are missing; in this way it can be assumed that the performance in the phase of transfer reflects the learning induced by the independent variable during the acquisition phase.

Some texts distinguish the second phase in true and proper retention and transfer (Weeks and Kordus, 1998), the latter into whether the subject is able to use the skills learned in a different context that is, if in its memory is constituted a more generalized pattern skill itself. For example, if a person has been exerted to pull darts at a certain distance from the target, in the retention test performed one or two days later, he will repeat the test at the same distance, while in the transfer test will ask the same task but at a distance greater or lesser than that exerted during the acquisition phase. The learning of motor skill is influenced by many factors, but a critical factor is certainly the availability of feedback that informs the learner about the success of its response (Salmoni, Schmidt and Walter, 1984; Winstein, 1991). The feedback is in fact used in the early stages of learning to generate or modify each subsequent pattern of movement, while later allows the comparison between the run and the correct reference movement. The term feedback means, literally, "feedback"; a feedback system is a system that has sensors that detect parameters of the output, makes the comparison between the movement performed and the desired one, and reports any error. The feedback thus informs the learner about the success of its response and is precisely why a critical factor for motor performance and learning (Salmoni, Schmidt and Walter, 1984). The information relating to the movement were divided into three categories:

1) Peripheral feedback or by countermovement of organs, mainly tactile and kinaesthetic;

2) Internal feedback or against central information, referred to by different authors also efferent copy or corollary discharge;

3) External feedback or counter on the effects of movement, that is, knowledge of the result; knowledge of result (CR) can be defined as information that represents the difference between the answers given and those ideals. An agreement in the literature regarding the motor behaviour is that some CR is necessary for learning a new motor response (Shea, Shebilske, \& Worchel, 1993).

The peripheral feedback and the knowledge of the result can be taken directly by the person who performs the task or supplied to it from an external source. From this point of view, Winstein (1987) respectively speak of intrinsic feedback and extrinsic feedback. The intrinsic feedback provides information both during and after the production and movement is inherent to information coming from the sensory receptors located in muscles, joints, tendons and skin, or by telocettori such as sight and hearing. In contrast, the extrinsic 
information feedback is coming from an external source which increases the intrinsic feedback. The external source may be the therapist or a device such as a biofeedback machine or a stopwatch. The greater the number of sensory channels through which the feedback is provided, the stronger will be the reference of correctness.

The potential of extrinsic feedback is shown by some studies that have shown that motor learning can take place in the absence of intrinsic feedback. In the classic study by Rothwell et al. (1982) parties, private afferents, were learning new motor tasks while receiving extrinsic visual feedback through the use of an oscilloscope. When visual feedback was removed, the person could perform the task but his decreased performance over time. The authors concluded that the feedback was necessary in some paragraphs to update the CNS about the success and especially the accuracy of the movement. Even the feedback extrinsic can be distinguished into information relating to the outcome of the action and information relating to the motor sequence that has produced the action itself. In the first case from the outside provides an enriched verbal feedback about the movement result, it has namely a form of Knowledge Result (CR). The CR provides information about the errors, so it provides a learner information on how to modify the movement in the next attempt. The Knowledge of Performance (CP) is instead a verbal feedback about the nature of the movement that can be provided only at the end of the movement. The research on the importance of feedback extrinsic motor learning has focused almost exclusively on CR. This preference is due essentially to the ease with which the CR can be obtained, quantified and manipulated under the experimental conditions. There are many potentially important variables for learning about the type of feedback provided to the person who learns a motor task and mode of administration of extrinsic feedback, by years of research. In research on the relationship between feedback and motor learning creates an experimental environmental condition in which the usefulness of the relevant feedback to the inherent result is minimized, then the feedback is reintroduced (typically in the form of CR) and examines its effects on the process learning (Winstein, 1991). So, an experimental protocol can closely mimic the conditions of a patient with sensory deficit that is unable to effectively use the intrinsic feedback for motor control and therefore has to rely on extrinsic feedback provided by the therapist. This research paradigm is based on the premise that the feedback extrinsic learning function of artificial tasks in the laboratory, in the same way it does the intrinsic feedback in real-life situations. Therefore it is believed that the processes facilitated by the use of extrinsic feedback in the laboratory, such as error correction or development of an internal reference of correctness, are similar to those processes facilitated by the use of intrinsic feedback sources under natural conditions, in which the CR is not available or is redundant. There are many parameters regarding the administration of the CR potentially able to influence the degree of motor learning, but most studies have focused on the relative frequency with which it is supplied, to the learner, knowledge of the result. With this term (relative frequency), is defined as the percentage of the trial for which is supplied the feedback during a practice session with respect to the total number of tests, while the absolute frequency term indicates the total number of trials for which it is provided that feedback (Winstein, 1991). For example, if the CP and / or CR are supplied every 2 tests in the framework of a 20-trial program, the absolute frequency information is 10 exposures, while the relative frequency is $50 \%$. The magnitude of the extrinsic feedback can be increased by increasing the number of trials and then increasing the absolute frequency, or by providing the information more often during the tests, thus increasing the relative frequency. There has been considerable debate about the frequency of CR and protocol of administration useful to optimize motor learning (Guadagnali Dornier \& Tandy, 1996; Swinnen, 1996). In general, the literature on the motor and the clinical practice protocols show a surprisingly constant that, during the practice in most tasks, almost every variation that increases the availability of information provided by the feedback (immediacy, accuracy, frequency, number of channels) benefits the performance and increases the percentage of improvement from one trial. If the performance benefits from these conditions, it is not said that these also promote learning and retention. From a theoretical point of view, two opposing assumptions 
about the optimal frequency of the CR have been suggested.

Early theories relating to information processing suggested that the CR is mainly used for motor learning in two complementary ways: a) the learner needs a CR to test the hypothesis of the correctness of the answer above, and b) any assumptions about the response that is tested contributes to a better memory of that response. In other words, after the completion of the test, the learner must formulate a hypothesis about its response, i.e. it must estimate with what success has stood the test. He must then verify the correctness of this hypothesis, and this can take place by the comparison with the CR received. On the basis of this comparison, it will develop a hypothesis response, or a plan, for the next trial. In agreement with this idea, Adams (1971) proposed that for motor learning subjects should use the CR to correct future answers based on information taken from previous answers. The strength of the memory that controls the response is developed as a function of the positive feedback produced by the response in relation to the CR, that is tested response hypothesis. Adams also noted that when knowledge the result is not provided, the learner can only reinforce what has been learned from previous responses with CR. Therefore, based on this perspective (Adams, 1971, 1987; Schmidt, 1975), it was expected that 100\% of the rate of CR should maximize the effects of training with regard to learning. In more recent theoretical perspectives about information processing, this hypothesis has been questioned. In fact, it was suggested that, in some way, when the person who has to learn is provided a high rate of $C R$, some formulations of the CR will be used to guide future responses. This type of case has been referred to as a guide hypothesis (Schmidt, 1991). One implication of the hypothesis guide is that when participants receive during the acquisition, a high rate of CR, they cannot use the additional memory processes or seeking additional sources of information that contribute to the development of memory. In contrast, when it is provided to the participants in a low frequency of the $\mathrm{CR}$, it is encouraged the commitment of an additional memory processes during the trial not guided by the knowledge of the result. These additional memory processes, in turn, promote the development of memory. Based on these latest perspectives on the use of CR for motor learning (Schmidt, 1991), it was stated that a low frequency of the CR, however, less than $100 \%$, can optimize motor learning effects long-term retention. Numerous studies have been conducted to compare the effect of a different relative frequency of CR on learning of a large number of motor skills, with results generally in favour of the hypothesis-driving. In most cases, in fact, it has been found that subjects who practiced in the task with a lower relative frequency of the feedback extrinsic best results obtained at a retention test. In fact, literature data to support the theory of the guide are plentiful. One of the first studies that supported the new theoretical perspectives was conducted in the early 90's by Winstein and Schmidt (1991) in which it sought to maximize the beneficial effects attributed to learning to practice in poor conditions relative frequency of $\mathrm{CR}$, through the manipulation of the test program with no $\mathrm{CR}$ and $\mathrm{CR}$ in a practice session. Two groups of subjects exerted a spatio-temporal movement pattern complex for 2 days in conditions of high (100\%) or moderate (50\%) relative frequency of CR. In this experiment, the number of trials (196 per day) was held constant between the groups, allowing covariate the relative frequency and absolute. In $50 \%$ of the group it was made a gradual reduction of the CR: in every day, the part of the tests with CR was relatively high at the beginning in practice (100\%), but it was gradually reduced toward the end of practice (25\%). After 2 days of practice, they were administered to each group a retention test after 5 minutes and after one day. The results showed that there was no difference in the degree of average error between the groups during the acquisition phase. In the immediate retention test without CR, the group with a 50\% CR obtained an error score slightly lower (8.5 versus 9.2) compared to the $100 \%$ group, and this difference was even more evident in the late retention test (10 against 12.1): for both groups, the performance got worse between the end of the acquisition and the retention test, but this deterioration was significantly higher in the group with $100 \% \mathrm{CR}$. These results were replicated in a second experiment (Winstein and Schmidt, 1990) in which he used the same of previous experiment CR program for a period of two days of practice, but in which the retention tests, executed the next day, It consisted of 12 
trials with CR provided after each test. Surprisingly, the 50\% group had significantly better performance compared to the $100 \%$ group of CR even in this retention test with $100 \% \mathrm{CR}$. The $50 \%$ reduction of the relative frequency of $\mathrm{CR}$ during the practice seemed therefore facilitate the development of a capacity of independent response from the particular surface characteristics of the practice and by retention conditions. The results of Winstein and Schmidt (1990) have been repeatedly confirmed by other authors comparing groups with different frequency of the CR (Nicholson \& Schmidt, 1991; Vander Linden et al., 1993; Weeks and Kordus, 1998). It is interesting to note that the results are similar even if the study population is elderly. Although there are obvious age-related changes regarding the cognitive and motor control, not much research has been conducted on the effect of age in learning motor skills. From the limited data available seems, however, that the ability to process the CR and the effects of this on learning motor are similar in young and elderly. Swanson and Lee (1992) examined the effects of different exposure programs to CR in young adults (20-23 years) and older adults (60-82 years). The results showed that there were differences between young adults and the elderly in the accuracy and constancy of performance when the CR was provided. Differences persisted in precisely the retention test without $\mathrm{CR}$, where, however, there were no differences in the variability. In addition, there were no age interactions with any of the variables related to CR. Similar results were reported by Wishart and Lee (1997). Using a trisegmentale task, in which each segment had specific timing goals, the authors compared three different manipulations of the relative frequency CR in young adults and seniors. The 3 conditions were 1) $100 \% \mathrm{CR}$, in which the CR related to all three segments was provided after each test, 2) $67 \% \mathrm{CR}$, in which the information progressively decreased in the different tests, and 3) $67 \% \mathrm{CR}$, in which the information was decreased in the various segments within each test. After 90 trials of the acquisition, all subjects performed tests retention, transfer and reacquisition. There were age-related differences regarding the accuracy and consistency of the movement in the acquisition and retention tests, but not in the transfer test. However, none of these differences was interacting with the manipulation of the frequency of the CR. Surprisingly, there was no effect attributable to the different ways in which information reduction. Evidence in support of the guiding theory also stem from data on the effectiveness of a summary CR (Gable, Shea, \& Wright, 1991; Suddon \& Lavery, 1962; Schmidt, Lange, \& Young, 1990; Carnahan et al., 1996) and a CR average (Yao, Fischman, \& Wang, 1994). A summary CR (summary KR) means to give information about the outcome of each test in a series of tests only after the whole series has been completed, instead of providing the feedback extrinsic after each single test. The CR average (averaged summary KR) is a variant in which the CR is presented as the mean value of the test series. In both cases it reduces the availability of the extrinsic feedback to guide the subsequent performance, availability, that according to the hypothesis-guide may discourage additional processing processes which in turn promote the development of memory. Even the results of the research on the influence of the simultaneous and continuous feedback also supported the theory, because the simultaneous feedback ensures an online guide, and has been repeatedly demonstrated that this hinders the learning of motor skill (Kohl and Shea, 1992). Support for rail theory is not, however, unanimous (Dunham and Mueller, 1993; Lee et al., 1990; Sidaway et al., 1991; Sparrow and Summers, 1992). Disparities in the results we have especially for more complex tasks studies (Guadagnoli et al., 1996). For example, Lai and Shea (1999) compared a group with $100 \%$ of FR with groups receiving feedback with FR less than 100\%, applied with different protocols, finding that the latter did not differ between them, but both were overcome by group 100\% to a retention test without CR. For this disparity of results, Guadagnoli and Kohl (2001) have recently proposed a suggestive explanation: what the subject is before receiving the CR, and how they will use it, are not independent of each other. In other words, if before receiving the feedback the subject elaborates a hypothesis about the performance just ended, he will use the CR to operate a comparison between its assumptions and the actually obtained result; of course, this comparison will not be possible for all trials for which he does not receive the information back. However, if the subject does not build a response hypothesis, the CR will be used in an entirely different way, namely, to mechanically drive the subsequent response. In 
fact, it is proven long ago that urge the subject to verbalize a subjective estimate of the error is a useful strategy to optimize learning. The first study that investigated the influence of an estimate of the performance by the learner a motor task was conducted by Swinnen et al. (1990). These authors compared the acquisition of skills for a group of subjects receiving $100 \%$ of CR after a short delay, with another group receiving $100 \%$ of CR instantly. However, the authors introduced the estimated variable, studying then 3 CR conditions: a) instantaneous CR, that is presented immediately after the completion of the movement, b) CR delayed, that is presented after an 8-second interval, and c) delayed CR with an interval 8 seconds, during which the subject was required to make an estimate of their performance. The results showed that, while during the acquisition phase there was no difference among the three groups, in an immediate retention test (after 10 minutes) and late (after 2 days) the group with immediate CR showed a sharp deterioration in the performance than the other two groups. In the late retention test, the group that during the practice had to make an estimate of the time taken had significantly better performance than the group with immediate CR, while the group with delayed CR had intermediate performance than the other two. Even a smaller interval (3.2 seconds) showed, in a second experiment, to have positive effects on learning compared to immediate feedback. Carnahan et al. (1996) have subsequently found that, for a manual tracking task, not only the presence of an interval between the end of the performance and extrinsic feedback is conducive to learning, but the greater the amplitude of the interval and more are also the positive effects. These effects are also observed at a transfer test, in which the tracking pattern was different from that exercised. In contrast, as expected, during the practice of the error it increased with the increase of the CR delay. Liu and Wrisberg (1997) have further examined the effects of a delay of the CR and the subjective evaluation of the shape of the movement in the acquisition and retention of a motor skill. During the acquisition, 4 groups of participants performed 60 trials of a task of precision launch, respectively with the following conditions: a) CR immediate, b) CR delayed, c) CR more immediate assessment of the shape and movement) CR delayed more evaluation of the movement form. The retention tests were designed to measure the accuracy of both the launch that the estimate of in the absence of visual feedback errors and were administered 5 minutes and 24 hours after the acquisition. The throwing accuracy was significantly higher during the acquisition, but significantly lower during the retention, in patients with $\mathrm{CR}$ immediate than those with delayed CR. However, the participants who evaluated the shape of the movement performed during the practice sessions provided regular launches with a significantly higher precision and with less error of judgment during the retention than other subjects. If the subjective assessment variable is a factor, it is understandable that a failure to control for this variable in the experimental protocol can lead to different results. For example, if you assume that the subjects of the study of Lai and Shea (1999) were spontaneously evaluating the correctness of its response, it would be possible to interpret their results in a different perspective: the arrangement of a larger number of trials that compare his hypothesis with the actual results (100\% relative of the feedback frequency) allows a greater number of comparisons and therefore a more durable trace in the memory. Under these circumstances, in fact, the degree of learning could be a function of how many times the answer hypothesis is tested. In most of the literature on the efficacy of a different frequency of the feedback extrinsic this aspect is not considered, however. Assuming that relative frequency error and subjective estimate could interact, and that this possible interaction may explain some conflicting data in the literature, Guadagnoli and Kohl (2001) have therefore conducted an interesting study to evaluate possible relationships between these two variables. The authors proposed with this re-evaluate the considerations on the relative frequency in the conditions in which participants are instructed to use different practical strategies that should, in theory, promote different uses of the CR for motor learning.64 young healthy subjects practiced a simple motor task of manufacturing a target isometric strength with muscles and flexor) should, or should not, make an estimate of the error made in the response just ended and b) receiving the CR after each trial (100\% relative frequency) or after every fifth trial (20\% relative frequency). The period of practice consisted of 10 sets of 15 repetitions of the task, performed during a single session, and a retention test, consisting of a series of 15 repetitions without CR 
and without estimation, was carried out to 24 hours apart. The analysis of the results showed that the group that received the $\mathrm{CR}$ with frequency of $100 \%$ and which was to subjectively estimate the error in the response made fewer errors at the retention test. The two groups receiving $20 \%$ of CR performed less well the retention test, no differences related to having or not having made the estimate, while the group receiving $100 \%$ of $C R$ and that it had no estimate showed performance the worst retention. These results are consistent with the expectation that learning is a function of the degree to which individuals use the CR to test the hypothesis about the answer. In fact, in conditions in which the subject's response includes the hypothesis, the CR will provide a verification of such a hypothesis, so a high frequency of the CR will optimize motor learning with respect to a low frequency of the $C R$. In contrast, a person cannot use the $C R$ to verify the hypothesis of response if the response does not include a hypothesis to be tested. Simple repetitive responses, which do not require participants to estimate the error of the answer, can deter people from creating response hypotheses, and in this case, theoretically, the CR can serve as a guide. This postulate is consistent with recent insights into the information processing of the $\mathrm{CR}$ is used as motor learning.

\section{PURPOSE OF THE STUDY}

The research on learning of a motor task, though usually conducted in healthy subjects, offers many suggestions about strategies that can be used to optimize recovery after CNS injury. Rehabilitation, in fact, must be seen as a learning process in pathological conditions. Among the different variables, the relative frequency with which it is supplied to the learner an extrinsic feedback about the outcome of the response (Result of Knowledge, CR) is certainly the most studied. The major evidence exists to support the hypothesis that a high frequency of the $C R$ during the period of practice interferes with learning, because the subject, in this case, uses the external information to guide the response and is not obliged to use an additional memory processes and to seek additional sources of information that further contribute to the development of memory. However, there is also evidence to the contrary, and it is postulated that these conflicting results may be explained considering possible interactions between the frequency of the $C R$ and subjective estimate of the error on the part of the learner (Guadagnoli and Kohl, 2001). For a long time, it is known, in fact, that request to the subject an estimate of the error made in a practice trial favours the motor skill fixation (Swinnen et al., 1990). It has been suggested (Guadagnoli and Kohl, 2001) and ask the person to formulate a hypothesis about the performance just ended (error estimate) may affect the way in which he will use CR came later: in this case the CR would be used not as a guide to the next performance, but rather to verify the correctness of the hypothesis formulated. If this hypothesis is correct, the person should benefit from a high relative frequency of feedback. The authors tested this hypothesis in learning a task of isometric force production with finger flexors. The results have provided some support to this theoretical approach, pointing out that the two variables (frequency of the CR and estimate) were not independent in their effects to a retention test (Guadagnoli and Kohl, 2001). The purpose of this study is to further test this hypothesis in the learning of a similar motor task, namely the production of a positive working target with the flexor muscles of the elbow in young healthy subjects.

\section{METHOD}

\section{Subjects}

They participated in the study 60 young healthy subjects, aged between 20 and 38 years (mean $24.1 \pm 3.2$ ), including 33 women and 27 men. The only inclusion criteria were:

1) The absence of pathologies of the central and peripheral nervous system;

2) Absence of musculoskeletal in the upper right limb level alterations. 
All subjects were informed of the purpose of the study and there were participating voluntarily.

\section{Procedure}

All subjects performed a single training session when learning of a simple motor task. The task consisted in producing a positive working target with the flexor muscles of the right limb elbow during isokinetic concentric contraction at a speed of $30^{\circ} / \mathrm{s}$.

For the study an isokinetic device REV 9000 (Technogym) was used. It is an apparatus which allows to perform diagnostic tests and exercises with both a continuous passive motion that in all types of muscle activities: isometric, isotonic and isokinetic (the latter in the two concentric and eccentric mode). The data relating to the torque, speed and position are stored with frequency of $100 \mathrm{~Hz}$. All the levers used to examine the different body segments are equipped with an anchor point mounted on a slide, in such a way that, during exercise, it will adapt to the joint fulcrum and are avoided harmful voltages to the articular structures. The application of belts and handles allows to obtain a good stabilization of the patient, so as to ensure the isolation of all the articular positions. The device is also equipped with a laser pointer light beam spot on the central pivot of the dynamometer, for centring with the utmost precision the joint fulcrum, and a system that eliminates the effects of the force of gravity due to the weight dell ' limb and the lever while performing the exercise. To have a good reliability of the force values of each subject, the system allows users to record with the utmost precision the position of all moving parts (backrest, the motor lock position, the lever position used, etc.), So that a given subject perform all trials with the joints affected in basically the same position. An earlier study has shown excellent reliability of the force measurements obtained with the REV 9000, with greater than $0.95 \mathrm{ICC}$. The working target to be learned was calculated as the one corresponding to one third of the average working expressed by 5 persons of the same sex of the same age $(23.5+1.5)$ during maximal isokinetic contraction at the same speed. None of the 10 subjects on which the ceiling work was detected later participated in the training. For the measurement of the maximum expressible work with the flexor muscles of the elbow, the subjects were asked to sit on the seat of the device, with the backrest tilted back $30^{\circ}$, and was adjusted the position of the support for the arm and the dynamometer in order to coincide the axis of the elbow joint, centred lateral epicondyle of the humerus, with the axis of rotation of the dynamometer. To make the movements of elbow flexion, the subject acting on the dynamometer lever gripping a handle with his right hand. Before performing the maximal strength test, the trunk and the pelvis of the subject they were stabilized with two crossed thoracic belts on the chest and with a cross-mail belt at the level of the pelvis, in order to avoid compensatory movements that could adversely affect the test result. After selecting the range of motion, for all that it was from $40^{\circ}$ to $130^{\circ}$ (measured between the lever arm and the dynamometer) and after acquiring the data relating to the weight of the limb, this gave rise to the test. To measure the maximum expressible work by flexor muscles of the elbow, they were performed 8 repetitions of the movement at a speed of $30 \% / \mathrm{s}$. The first three repetitions, used as heating and to familiarize the subject with the machine, they were carried by the subject with the instruction to exert a sub-maximal strength, that is to exert a force high enough without arriving at the maximum available force. These first heating repetitions were performed by the subject without a predetermined interval between one repetition and the next. After completing the three repetitions sub-ceilings is passed, after a rest interval of 30 seconds, at five repetitions used to measure the maximum work. For five repetitions ceilings the subject was instructed to perform elbow flexion movement exerting maximum force available at any point of the range. The repeats were separated by an interval of $5 / 2$, in order to avoid effects of muscle fatigue. When the test was recorded the highest labour expressed in joules, i.e. the work performed during the best repetition. So is the average of the values obtained was calculated in the five subjects of the same sex and the target to be learned has been established as corresponding to a third of that average value. Based on the results obtained on 10 subjects who carried out the maximal contractions, the motor task to be learned was as follows: for males, 
the production of a work of $23 \mathrm{~J}$, for female subjects, producing a work of $15 \mathrm{~J}$. All study participants were then exercised the task to be learned during a single training session, consisting of 15 sets of 10 repetitions of the task, for a total of 150 reps. The interval between repetitions of a same series was about 7 seconds, while that between the different series was about 4 minutes. During training, the general location of the subject and the dynamometer and the excursion movement of the elbow were the same used for the evaluation work of the ceiling, with the only difference that during the training were not applied to the belts to stabilize the trunk, since it was not required to subject a maximal contraction. However, before starting the training, were recorded for each subject the height and antero-posterior position of the dynamometer, the position of the support for the arm and the position of the handle, so as to perform the subsequent retention test under the same conditions. To study the influence of a different relative frequency CR, of a different frequency error and subjective estimate of a possible interaction between these two variables, the subjects were randomly assigned to four groups:

- Group A(100\% FR no SS): the subjects (mean age $24 \pm 3.2$ ) assigned to this group received the CR after each attempt (100\% FR), with a delay of about 3 seconds from the end of each test; Immediately after the completion of each repetition, these subjects were asked to perform a simple mental calculation (mathematical operation), while it was not them ever required to make a subjective estimate of the positive work produced during the trial just ended (no SS);

- Group B $(100 \%$ FR + SS): the subjects (mean age $23.8 \pm 3.4)$ assigned to this group also they received the CR after each attempt (100\% FR), with a delay of about 3 seconds from the end of each test; Immediately after the completion of each repetition, before providing the $\mathrm{CR}$, these subjects were asked to give a subjective judgment about the work done during the test (+ SS);

- $\quad$ group (20\% FR no SS): these subjects (mean age $23.8 \pm 1.9)$ were receiving feedback twice during each set of ten reps, and more specifically after the fifth and the tenth of each set trial ( $20 \% \mathrm{FR})$; also in this case the information was supplied with a delay of 3 seconds compared to the end of the test; these subjects (such as those of group A) was never required to give a personal opinion about the performance just ended (no SS) and immediately following completion of each repetition, as an element distracting, were invited to perform a simple mathematical operation;

- D group (20\% FR + SS): in this case the extrinsic feedback was provided to the subjects (mean age $25 \pm 4.1$ ) with a relative frequency of $20 \%$, with the same group $C$ mode, while a subjective estimate was request to the subject immediately after the conclusion of each test. The day after the training session, all subjects performed a retention test, consisting of a single set of 10 repetitions. During the retention test all groups were equalled: the subjects in fact, regardless of the training group, during this test did not receive any information about the outcome of the performance just ended, and it was not ever asked them or to make a subjective estimate, or to perform a mental calculation.

\section{Data analysis}

For each series it was calculated error average compared to the target done during each repetition and this value was then expressed as a percentage compared to the target. It is the mean and the standard error was then calculated for each group. The statistical analysis was performed only for the data related to the first series of practice and testing of retention. For the comparison between the groups at the retention test it was used analysis of variance, before and after adjustment for the initial conditions. 


\section{RESULTS}

It was illustrated the behaviour of the variable being studied in the course of the acquisition phase and the retention test for the four groups of subjects (100\% FR no SS, 100\% FR + SS, 20\% FR no SS, and 20\% FR + SS). In all groups there is a progressive reduction of the error during the practice, a result which was retained only in part to the retention test. Data shows the mean and the standard error for the four groups during the first series of practice, considered as a baseline, and during the retention test.

Group Error

Baseline Retention

$100 \%$ FR no SS $3.059+2.216+0.2540 .355$

$100 \%$ FR + SS $2.953+2.329+0.2520 .312$

$20 \%$ FR no SS $4.267+2.165+0.7970 .314$

$20 \% \mathrm{FR}+\mathrm{SS} 4.007+1.283+0.5720 .094$

The analysis of variance revealed no significant differences between the groups in the provision at baseline ( $p=0.1884$, see next data), even if the average error was significantly higher in Groups 3 and 4 with respect to the groups 1 and 2. The comparison between the groups at the retention test also it indicated that the differences related to the type of training were not significant, although it noted a trend to a better performance of the group 4 ( $p=0.073$ ). If the analysis was performed after adjustment for the initial conditions, the difference between groups was significant:

\section{$\mathrm{Fp}$}

Error T 11.650 .1884

Ret 2:45 0.0735 Error

Ret error after

adjustment for T1 2.790 .0491

\section{DISCUSSION AND CONCLUSIONS}

The present study was designed to assess how they interact the two variables of the FR low external feedback and error subjective estimate by the person who practices the task. From a theoretical point of view (Guadagnoli and Kohl, 2001), it is possible to assume that, if the subject needs to formulate a SS before receiving the $C R$, he will benefit from a high $F R$ of $C R$, because in this way may make a many comparisons between their own assumptions about the response and the results actually achieved. On the contrary, if the SS is not formulated, the subject will benefit from a FR of the CR low, because it will thus be encouraged to engage in additional memory processing processes, which in turn promote the development of memory trace. With a high FR feedback, some formulations of the CR will be used, therefore, to guide future responses (Schmidt, 1991; Schmidt and Bjork, 1992). The results obtained by us, for a similar motor task, do not support this hypothesis and are in contrast with those reported by Guadagnoli and Kohl (2001). In fact, among our subjects, only those assigned to the group $4(20 \%$ FR + SS) differed significantly from the others. The performance of the other three groups at the retention test was very similar. These results assume that the most important variable in all cases, the reduction of the FR. In fact, the subjects of the group 2 (100 FR + SS) did not differ from the others, indicating that the request to formulate a hypothesis about its response posed no facilitation in the presence of a high FR of the external feedback. These subjects, presumably, they used the information supplied from the outside to drive the answer in the next trial to an extent more or less similar to what happened for the subjects in Group 1 (no SS 100 FR). The demand for a SS does not seem 
to constitute, in itself, an effective strategy to optimize learning, in the presence of FR high, which is obviously a very important negative factor, as has been shown in numerous studies (Winstein and Schmidt, 1990; Nicholson \& Schmidt, 1991; Vander Linden et al., 1993 Week \& Kordus, 1998).The fact that the subjects of group $3(20 \%$ FR no SS) no they were different from the others could be explained by considering that in our protocol the condition no SS was not simply the lack of a facilitating factor potential but rather the introduction of a disorder . In these subjects, in fact, instead of the formulation of an estimate about its own error, it was required to perform a mental calculation immediately after the completion of the trial, in a phase that is to say in which the subject had to engage in memory processing processes. From this point of view our data cannot be fully compared with those reported by Guadagnoli and Kohl (2001). It can be assumed that when the subjects were asked to solve a simple mathematical operation at this crucial stage, the disturbing effect was such that it completely negates the advantage resulting from a lower FR external feedback. In light of this consideration, it is not possible to determine in fact, according to our results, whether the introduction of the request to formulate a subjective assumption about their own performance accurately depicted a facility for learning motor tasks we use. Indeed, the fact that the low association FR + SS has led to better results than the low condition FR no SS could be due simply to the fact that the low condition FR no SS was in fact a condition in which the low-FR was associated with a disturbing factor (execution of mental calculation). The literature, however, shows many evidences that the subjective estimate request favours the motor skill fixation practiced (Swinnen et al., 1990; Carnahan et al., 1996; Liu and Wrisberg, 1997). In all these works, in fact, the comparison was made between the SS or SS conditions, without introduction of a disorder. It is in our view the relevant since the introduction of mental calculation for no SS groups has proved a very powerful disturbing factor as to nullify any advantage consists of a low-FR. Probably it is not always aware of how important it is to avoid disturbing elements in the phase immediately following the completion of a motor task. This has important implications for the physical therapist who works with patients neurologically. Many might in fact be the potential environmental disturbances, such as noise and crowding of the gym, phones, music at high volume, the physical therapist behaviour. Also, you have to stress the fact that the completion of a motor performance to be learned does not exhaust the mental exercise that the subject has to perform. Do not check the requests addressed to the subject is in the unwinding phase of the task, both in the one immediately after, it may therefore influence in a negative way the effects of rehabilitation.

\section{REFERENCES}

Adams, J. A. (1971). A closed-loop theory of motor learning. Journal of motor behavior, 3(2), 111-150. https://doi.org/10.1080/00222895.1971.10734898

Adams, J. A. (1987). Historical review and appraisal of research on the learning, retention, and transfer of human motor skills. Psychological bulletin, 101(1), 41. https://doi.org/10.1037//00332909.101.1.41

Carnahan, H., Vandervoort, A. A., \& Swanson, L. R. (1996). The influence of summary knowledge of results and aging on motor learning. Research Quarterly for Exercise and Sport, 67(3), 280-287. https://doi.org/10.1080/02701367.1996.10607955

Gable, C. D., Shea, C. H., \& Wright, D. L. (1991). Summary knowledge of results. Research quarterly for exercise and sport, 62(3), 285-292. https://doi.org/10.1080/02701367.1991.10608725

Guadagnoli, M. A., \& Kohl, R. M. (2001). Knowledge of results for motor learning: relationship between error estimation and knowledge of results frequency. Journal of motor behavior, 33(2), 217-224. https://doi.org/10.1080/00222890109603152

Guadagnoli, M. A., Dornier, L. A., \& Tandy, R. D. (1996). Optimal length for summary knowledge of results: the influence of task-related experience and complexity. Research Quarterly for Exercise and Sport, 67(2), 239-248. https://doi.org/10.1080/02701367.1996.10607950 
Kohl, R. M., \& Shea, C. H. (1992). Pew (1966) revisited: Acquisition of hierarchical control as a function of observational practice. Journal of Motor Behavior, 24(3), 247-260. https://doi.org/10.1080/00222895.1992.9941620

Lai, Q., \& Shea, C. H. (1999). Bandwidth knowledge of results enhances generalized motor program learning. Research quarterly for exercise and sport, 70(1), 79-83. https://doi.org/10.1080/02701367.1999.10607734

Liu, J., \& Wrisberg, C. A. (1997). The effect of knowledge of results delay and the subjective estimation of movement form on the acquisition and retention of a motor skill. Research Quarterly for Exercise and Sport, 68(2), 145-151. https://doi.org/10.1080/02701367.1997.10607990

Nicholson, D. E., \& Schmidt, R. A. (1991, September). Scheduling information feedback to enhance training effectiveness. In Proceedings of the Human Factors Society Annual Meeting (Vol. 35, No. 19, pp. 1400-1402). Sage CA: Los Angeles, CA: SAGE Publications. https://doi.org/10.1177/154193129103501913

Salmoni, A. W., Schmidt, R. A., \& Walter, C. B. (1984). Knowledge of results and motor learning: a review and critical reappraisal. Psychological bulletin, 95(3), 355. https://doi.org/10.1037//0033$\underline{2909.95 .3 .355}$

Schmidt, R. A. (1975). A schema theory of discrete motor skill learning. Psychological review, 82(4), 225. https://doi.org/10.1037/h0076770

Schmidt, R. A. (1991). Motor learning \& performance: From principles to practice. Human Kinetics Books.

Schmidt, R. A., \& Bjork, R. A. (1992). New conceptualizations of practice: Common principles in three paradigms suggest new concepts for training. Psychological science, 3(4), 207-218. https://doi.org/10.1111/j.1467-9280.1992.tb00029.x

Schmidt, R. A., Lange, C., \& Young, D. E. (1990). Optimizing summary knowledge of results for skill learning. Human Movement Science, 9(3-5), 325-348. https://doi.org/10.1016/0167-9457(90)90007$\underline{z}$

Shea, C. H., Shebilske, W., \& Worchel, S. (1993). Motor learning and control. Prentice Hall.

Suddon, F. H., \& Lavery, J. J. (1962). The effect of amount of training on retention of a simple motor skill with 0 -and 5 -trial delays of knowledge of results. Canadian Journal of Psychology/Revue canadienne de psychologie, 16(4), 312. https://doi.org/10.1037/h0083258

Swinnen, S. P. (1996). for Motor Skill Learning: A Review. Advances in motor learning and control, 37.

Swinnen, S. P., Schmidt, R. A., Nicholson, D. E., \& Shapiro, D. C. (1990). Information feedback for skill acquisition: Instantaneous knowledge of results degrades learning. Journal of Experimental Psychology: Learning, Memory, and Cognition, 16(4), 706. https://doi.org/10.1037//02787393.16.4.706

Vander Linden, D. W., Cauraugh, J. H., \& Greene, T. A. (1993). The effect of frequency of kinetic feedback on learning an isometric force production task in nondisabled subjects. Physical Therapy, 73(2), 79-87. https://doi.org/10.1093/pti/73.2.79

Weeks, D. L., \& Kordus, R. N. (1998). Relative frequency of knowledge of performance and motor skill learning. Research Quarterly for Exercise and Sport, 69(3), 224-230. https://doi.org/10.1080/02701367.1998.10607689

Winstein, C. J. (1987). Motor learning considerations in stroke rehabilitation. Stroke rehabilitation: the recovery of motor control. Chicago: Year Book Medical, 109-34.

Winstein, C. J. (1991). Knowledge of results and motor learning-implications for physical therapy. Physical therapy, 71(2), 140-149. https://doi.org/10.1093/pt/71.2.140

Winstein, C. J., \& Schmidt, R. A. (1990). Reduced frequency of knowledge of results enhances motor skill learning. Journal of Experimental Psychology: Learning, Memory, and Cognition, 16(4), 677. https://doi.org/10.1037//0278-7393.16.4.677 
Wishart, L. R., \& Lee, T. D. (1997). Effects of aging and reduced relative frequency of knowledge of results on learning a motor skill. Perceptual and motor skills, 84(3), 1107-1122. https://doi.org/10.2466/pms.1997.84.3.1107

Yao, W. X., Fischman, M. G., \& Wang, Y. T. (1994). Motor skill acquisition and retention as a function of average feedback, summary feedback, and performance variability. Journal of motor behavior, 26(3), 273-282. https://doi.org/10.1080/00222895.1994.9941683

\section{(c) (i) $\ominus$}

This work is licensed under a Attribution-NonCommercial-NoDerivatives 4.0 International (CC BY-NC-ND 4.0). 